\title{
The FIT therapy for the treatment of musculoskeletal and neurological disorders related symptoms: A retrospective observational study
}

\author{
Beniamino Palmieri', Maria Vadalà ${ }^{2}$, Carmen Laurino ${ }^{3}$ \\ ${ }^{1,2,3}$ Department of General Surgery and Surgical Specialities, University of Modena and Reggio Emilia. Largo del Pozzo \\ 71, 41124 Modena (MO). Second Opinion Medical Network, Modena (MO), Italy
}

Background: Far Infrared Waves (FIW) with frequency range among few hundreds gigahertz $(\mathrm{GHz})$ and few terahertz $(\mathrm{THz})$ display some positive effects on neurological and musculoskeletal disorders. Aims and Objectives: Our retrospective observational study describes the benefits observed by the administration of an infrared -trapping and focusing plaster (FIT-PATCH) in patients affected by different musculoskeletal and neurological symptoms. Materials and Methods: 100 patients were retrospectively reviewed through the Second Opinion Medical Network. Each patient sticked a FIT-PATCH every 5 days for 1 month upon the skin in the painful/inflamed area. Quality of life (QOL) assessment was evaluated by the Short Form-36 (SF-36) health survey questionnaire before starting the treatment and after the fourth week and the Visual Analogue Scale (VAS) completed the pre-post treatment subjective pain record. Results: SF-36 showed significantly improvement of the pre-post treatment scores $(P<0.03)$, pain score $(P<0.02)$, general health score $(P<0.03)$ and in the emotional component scores $(P<0.03)$. The VAS pain score was either improved $(\mathrm{P}<0.02)$ after plaster administration. No side effects or allergenic skin reactions were reported along the study. Conclusions: The FIT patches improved the symptoms probably through a mechanism involving the sebaceous/sweat glands system spreading the fit irradiating infrared $\mathrm{THz}$ waves energy, to the surrounding tissues with both a thermal and electromagnetic putative effect. The occlusive mechanism of the patch on the skin surface increasing the local thermic gradient cannot be adequately supposed to be therapeutically effective, because the FIT patch locally applied induces haemodynamic microvascular modification, not only locally but also remote. Thus, the intrinsic chemical properties of the FIT formulation absorbs and concentrates the endogenous infrared waves amplifying and reverberating them into the underlying tissues near and far, through low- $\mathrm{Q}$ factor helical antennas of the sweat glands spreading the energy all over the body. Hence, the claim is that fit basically absorbs and concentrates the endogenous infrared waves into the affected districts and accelerates symptoms recovery.

Key words: Infrared therapy; Electromagnetic; Neurological; Musculoskeletal; Patch; Life quality; FIT; Disease
Access this article online Website:

http://nepjol.info/index.php/AJMS DOI: 10.3126/ajms.v10i5.21230 E-ISSN: $2091-0576$ P-ISSN: $2467-9100$

\section{INTRODUCTION}

Musculoskeletal and neurological disorders include a wild spectrum of diseases with different related symptoms in terms of entity, duration, prognosis that negatively influence life quality of patients. Different therapeutic approaches are available, such as pharmacological treatments, dietary supplementation, surgery, manual and electro - medical physiotherapy. A new non-invasive strategy for the treatment of these pathologies, especially in the early stages of the 
disease, consists in the application of non-drugs patches in the affected body area. The system essentially consists of two elements: a non-woven polypropylene fabric and an acrylic adhesive. A flexographic printing is performed on the fabric. A percentage of titanium metal dioxides of different sizes, called AT5.05, is inserted in the pigments, which reflect the Far Infrared Waves (FIW) that the body emits, such as has been demonstrated by some tests performed at the CNR. Technology is covered by industrial secrecy. The electromagnetic waves with the frequency range spanning from few hundreds of gigahertz $(\mathrm{GHz})$ to few terahertz $(\mathrm{THz})$ are known as terahertz waves that correspond to the FIW. The wavelength of the radiation in the $\mathrm{THz}$ range corresponds to a range between $1 \mathrm{~mm}$ and $0.1 \mathrm{~mm}$ (or $100 \mu \mathrm{m}$ ) and it is not visible to the human eye, since it has a wavelength much greater than that perceived by it (visible spectrum). These waves can penetrate through wide variety of materials such as plastics, papers and cloths making them an alternative to X-rays in many applications such as security screening and non-destructive testing and evaluation ${ }^{1}$. Despite these developments, a thorough study of the interaction of the FIW with human skin has yet to be undertaken. More worryingly, evidence of non-thermal biological effects of electromagnetic energy in the $\mathrm{GHz}$ range is beginning to emerge $\mathrm{e}^{2-5}$, suggesting a potential mechanism of action in cellular communications. Therefore, it is crucial to understand the possible biological effects induced by $\mathrm{THz}$ waves on human beings. When the wavelength of electromagnetic waves is comparable to the dimension of biological structures, it is important to know the electromagnetic wave interaction with these structures and to consider possible resonating behaviour.

This retrospective observational study investigates the effects of an infrared -trapping and focusing plaster [(FIT-PATCH), D.FENSTEC s.r.l. Altavilla Vicentina (VI) ITALY)] in a clinical setting of different musculoskeletal and neurological symptoms.

\section{MATERIALS AND METHODS}

\section{Patients}

We reviewed medical records (electronic or paper-based) of 100 patients that had appealed to our "Second Opinion Medical Consulting Network", Medical Centre (Modena, Italy), between September 2017 and March 2018, because of musculoskeletal and neurological disorders. Patients were aged over 29 years and were affected at least 6 months by different musculoskeletal ( $\mathrm{n}=50$ patients) and neurological disorders ( $\mathrm{n}=50$ patients) predominantly in the neuro - musculoskeletal vegetative background with an attested related symptomatology (local pain) and diagnosis. The Second Opinion Medical Network is a consultation referral web and Medical Office System recruiting a wide panel of real-time available specialists, to whom any patient affected by any disease or syndrome and not satisfied with the diagnosis or therapy, can apply for an individual clinical audit ${ }^{6}$. Due to the doctor-patient communication gap, most of the patients usually wander around the medical websites looking for proper answers to their health problems. However, this search often becomes compulsive and obsessive and is frequently ambiguous and frustrating. Palmieri et $\mathrm{al}^{7}$. describe this borderline or even pathological behaviour as the "Web Babel Syndrome" - a psychological imbalance affecting young and elderly patients, especially those with multiple synchronous diseases who receive from their caregivers heterogeneous and misleading information or advice, including confused, contradictory statements and prescriptions ${ }^{8}$. To deal with this problem, the Second Opinion Network aims to be a useful "problem-solving" support revisiting each diagnostic and therapeutic step and properly re-addressing tailored treatments and prognoses, as well as preventing unnecessary investigational procedures and unhelpful and expensive medical and surgical interventions ${ }^{6}$.

All patients used pharmacological treatments, dietary supplementation, surgery, manual and electro - medical physiotherapy especially for the management of pain and stiffness drawing partial benefit from them. The most used drugs included analgesics, non-steroidal antiinflammatory drugs (NSAIDs), simple, and combination opioid analgesics, barbiturates, lidocaine, corticosteroids, hyaluronic acid, muscle relaxing drugs and vitamin B12 at high doses. However, their use is very often related to light, moderate and severe side effects (e.g. hypersensitivity reactions, sensation of pressure or constriction, flushing to face and in the thoracic region, asthenia, myalgia, drowsiness, insomnia, restlessness, vertigo, gastrointestinal symptoms, skin rashes, nausea, vomiting, etc.), as well as being limited by frequent drug interactions and contraindications. Dietary supplementation included herbal products and nutraceutical supplementations, while electromedical physiotherapy was conducted by laser therapy, iontophoresis, magneto therapy, electrostimulation, etc.

In our clinical practice, we suspended (wash out) previous treatments for a period of 2 weeks, and then the FIT therapy was administered.

The selected patients were informed, via individual interview, and informed consent previously approved by the Local Institutional Review Board under the Helsinki Declaration.

\section{Study design}

We conducted a retrospective observational study for 1-month duration. During this period, patients were 
educated to apply a FIT therapy patch every 5 days and then to replace the patch with another one until the end of the study period (Photos 1-6).

Different typologies of FIT therapy patches were available according to the anatomical area to be treated and every patient used the most appropriate patch according to the

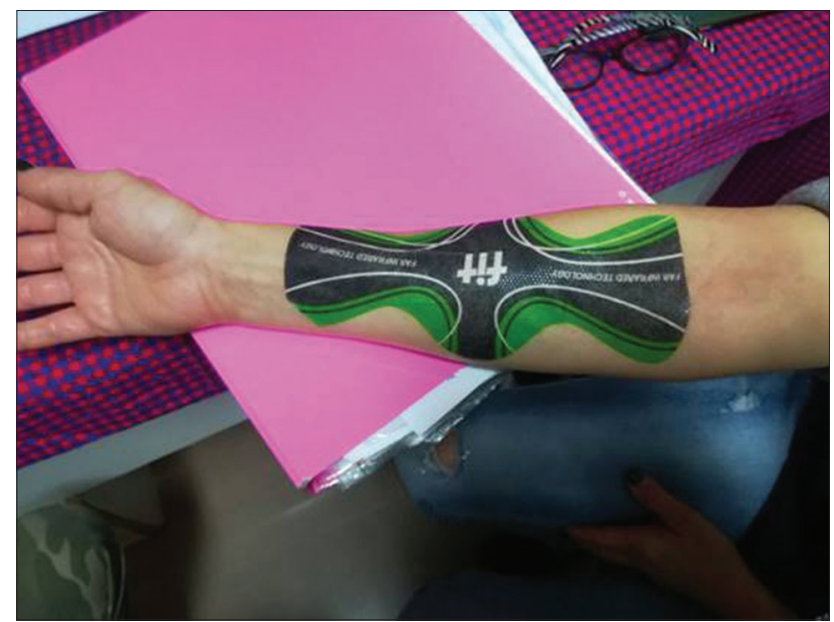

Photo 1: A 50 years old male patient with intense pain on inferior forearm

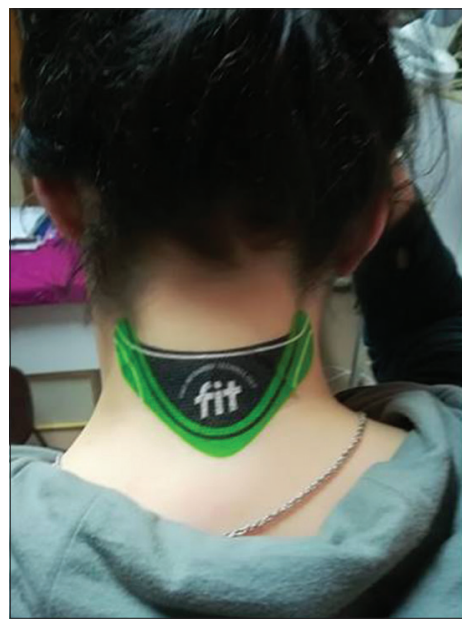

Photo 2: A 29 years old female patient with neck pain

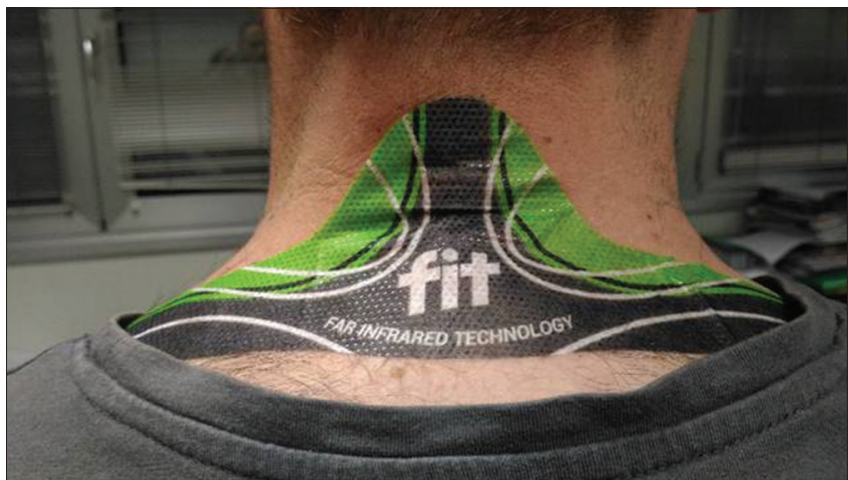

Photo 3: A 35 years old male patient with cervical pain clinical examination by the medical doctor. The available formats were "universal", "cervical", "shoulder", "elbow", "lumbar", "knee" and "ankle". All the patches were waterresistant, so it was possible to have shower wearing it.

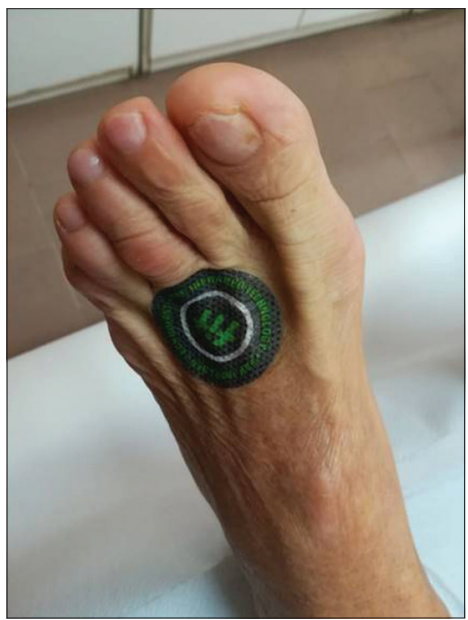

Photo 4: A 70 years old male patient with foot pain

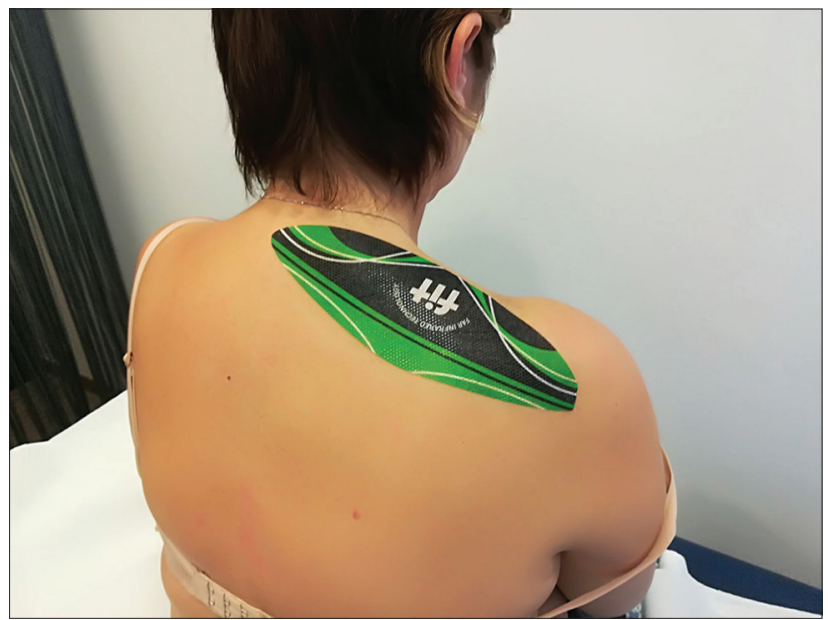

Photo 5: A 45 years old female patient with intense pain on shoulder

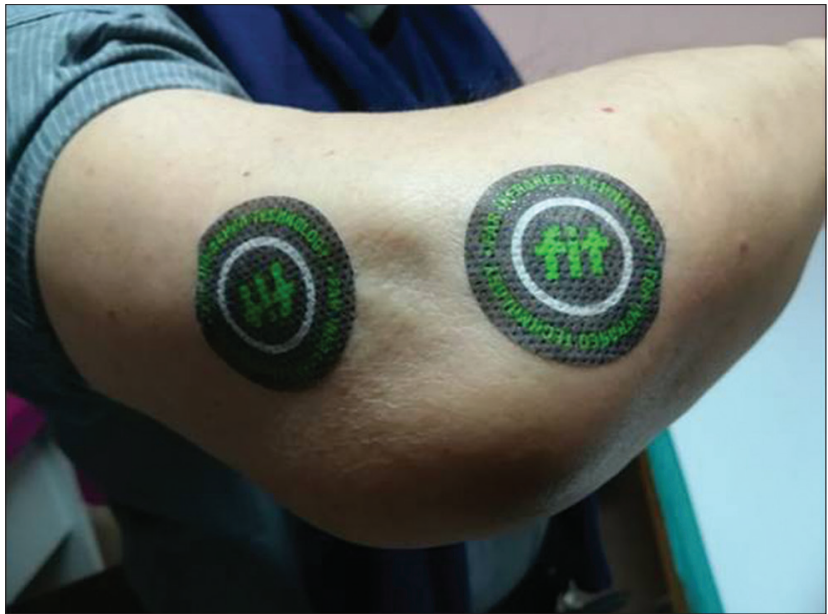

Photo 6: A 37 years old male patient with local elbow pain

Asian Journal of Medical Sciences | Sep-Oct 2019 | Vol 10 | Issue 5 
The first clinical examination consisted in the evaluation of the physical status of the patient. Tolerability and adverse effects were assessed weekly during the treatment period through direct contact (email or telephone) with the patients or their parents.

Quality of life (QOL) assessment was performed using the Short Form-36 (SF-36) health survey questionnaire before starting the treatment and after the fourth week. The questionnaire measures health-related quality of life in eight domains: physical functioning $(\mathrm{A})$, role limitations due to physical health (B), pain (C), general health (D), energy/fatigue (E), social functioning $(\mathrm{F})$, role limitations due to emotional problems $(\mathrm{G})$ and emotional well-being $(\mathrm{H})$. The percentage scores range from $0 \%$ (lowest or worst response) to $100 \%$ (highest or best possible response) ${ }^{9}$.

Visual Analogue Scale (VAS) was adopted for pain assessment before starting the treatment and after the fourth week $(0-100 \mathrm{~mm} ; 0 \mathrm{~mm}=$ minimum pain; $100 \mathrm{~mm}$ $=$ maximum pain)

\section{Statistical analysis}

The statistical analysis was performed using the MannWhitney test (continuous variables not normally distributed) and the chi-squared test (categorical variables). A commonly used measure of linear correlation, the Pearson correlation

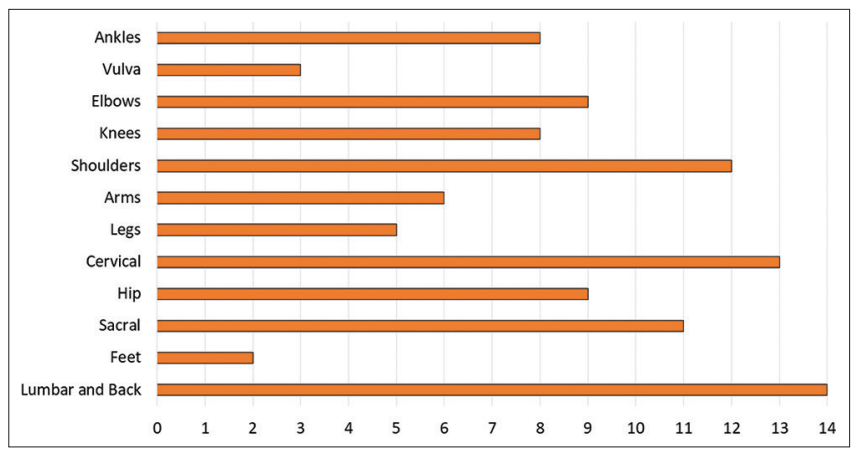

Figure 1: Body areas affected by local symptomatology underwent that were treated by FIT therapy

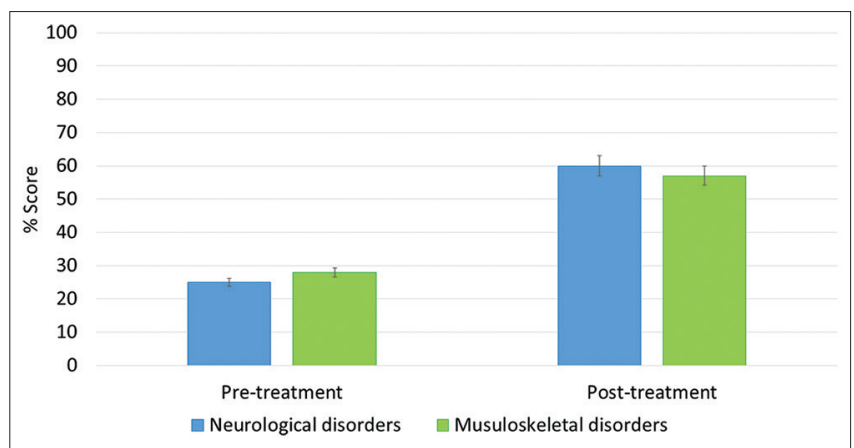

Figure 2: Significant improvement $(P<0.03)$ in the \% score of the physical functioning item of the SF-36 questionnaire after 1-month FIT therapy coefficient, denoted by $r$, was reported. Statistical significance was set at a $\mathrm{P}$ value $<0.05$, and all data and graphics were analysed using the $\mathrm{R}$ software, version 3.1.2.

\section{RESULTS}

Patients. 100 patients [ 50 women and 50 men, aged between 29 and 86 years old (mean age 59 years old)] followed the treatment for 3 months. 20 women $(40 \%)$ were affected by neurological disorders and $30(60 \%)$ by musculoskeletal disorders, while 30 men $(60 \%)$ were affected by neurological disorders and 20 men (40\%) by musculoskeletal disorders.

Most frequently, the locally treated anatomic area included the cervical - lumbosacral spine tract, the coxofemoral, the femoro-tibial, the acromio-clavicular joints and arms, due to symptomatic epicondylitis, coxo-arthrosis, arthritis, and hernias. Figure 1 shows medicated body areas and number of affected patients.

SF-36 revealed a significant improvement in the physical component scores (A-B; $\mathrm{P}<0.03)$, pain score $(\mathrm{C} ; \mathrm{P}<0.02)$, general health score $(\mathrm{D} ; \mathrm{P}<0.03)$ and in the emotional component scores $(\mathrm{G}-\mathrm{H} ; \mathrm{P}<0.03)$ after treatment (Figures 2-5 and Figures 8-9). No significant differences in energy/ fatigue $(\mathrm{E})$ and social functioning $(\mathrm{F})$ scores were found

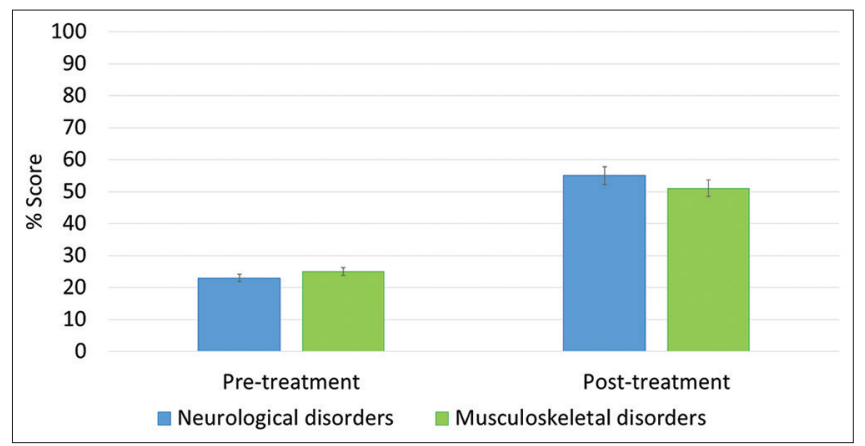

Figure 3: Significant improvement $(P<0.03)$ in the \% score of the role limitations due to physical health item of the SF-36 questionnaire after 1-month FIT therapy

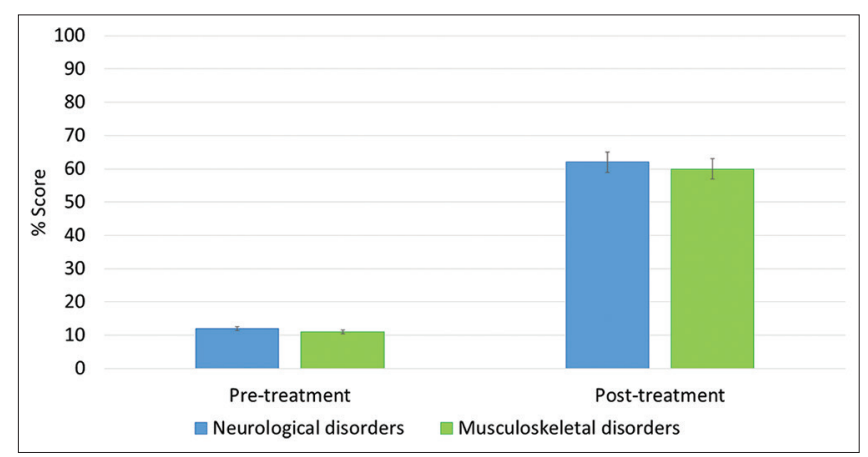

Figure 4: Significant improvement $(P<0.02)$ in the \% score of body pain item of the SF-36 questionnaire after 1-month FIT therapy 
after the fourth week of treatment $(\mathrm{P}>0.05)$ (Figures 6-7). A significant improvement in VAS pain score was also found $(\mathrm{P}<0.02)$ after treatment (Figure 10). A perception of heat was reported in about $20 \%$ of patients, and was considered beneficial in order to resolve the symptoms. The treatment with FIT therapy patches was well tolerated by all the patients. Any adverse effects have occurred.

\section{DISCUSSION}

This retrospective observational study evidences that the treatment of patients with neurologic and musculoskeletal

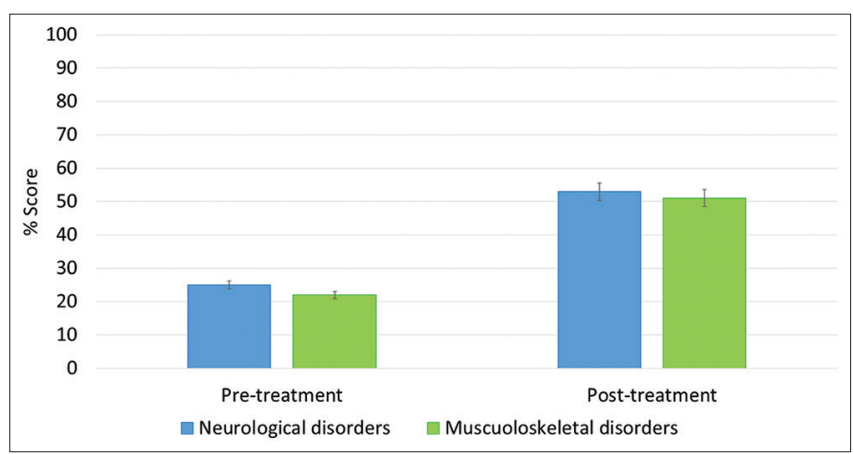

Figure 5: Significant improvement $(P<0.03)$ in the \% score of general health item of the SF-36 questionnaire after 1-month FIT therapy

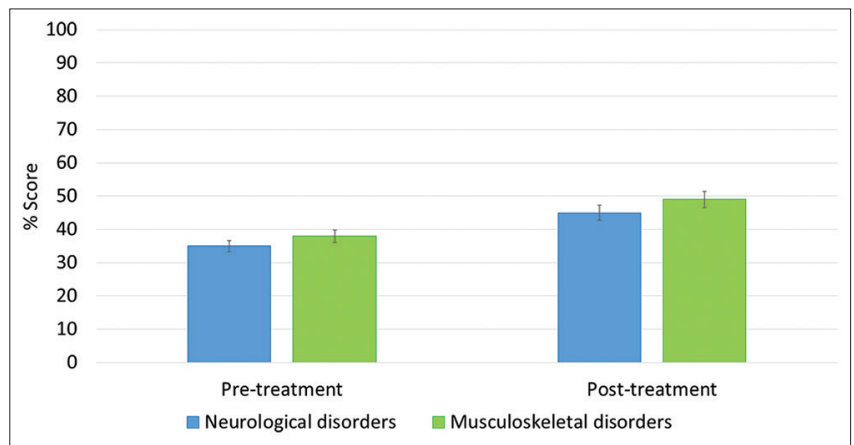

Figure 6: No significant improvement $(P>0.05)$ in the \% score of energy/fatigue item of the SF-36 questionnaire after 1-month FIT therapy

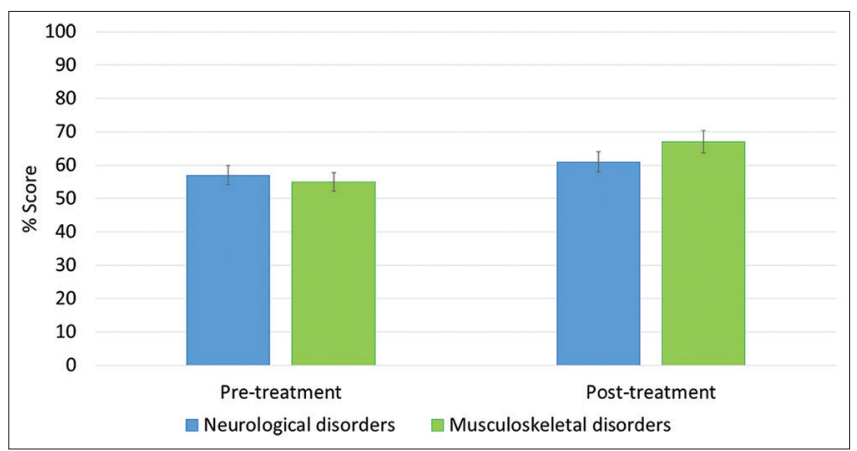

Figure 7: No significant improvement $(P>0.05)$ in the \% score of social functioning item of the SF-36 questionnaire after 1-month FIT therapy disorders with FIT therapy patches improved pain, physical and emotional status and related role limitations by alleviating the symptomatology. Neither adverse events nor skin intolerance to the patch were detected along the trial.

Although a light perception of heat that has been reported in about $20 \%$ of patients, we believe that the antalgic action is not heat-dependent. It remains to be defined that the invoked mechanism of action is not due to the simple occlusive mechanism of the patch, which prevents the transpiration sebaceous/sweat increases the local temperature, generating a gradient that has therapeutic effects.

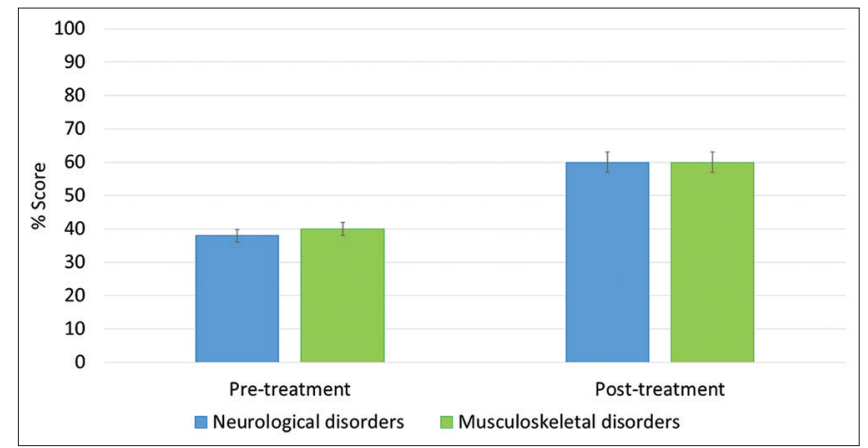

Figure 8: Significant improvement $(P<0.03)$ in the \% score of role limitations due to emotional problems item of the SF-36 questionnaire after 1-month FIT therapy

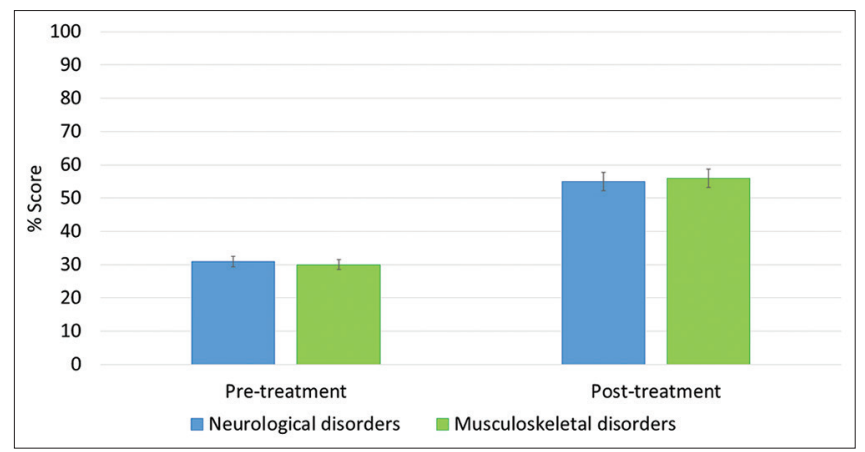

Figure 9: Significant improvement $(P<0.03)$ in the \% score of emotional well-being item of the SF-36 questionnaire after 1-month FIT therapy

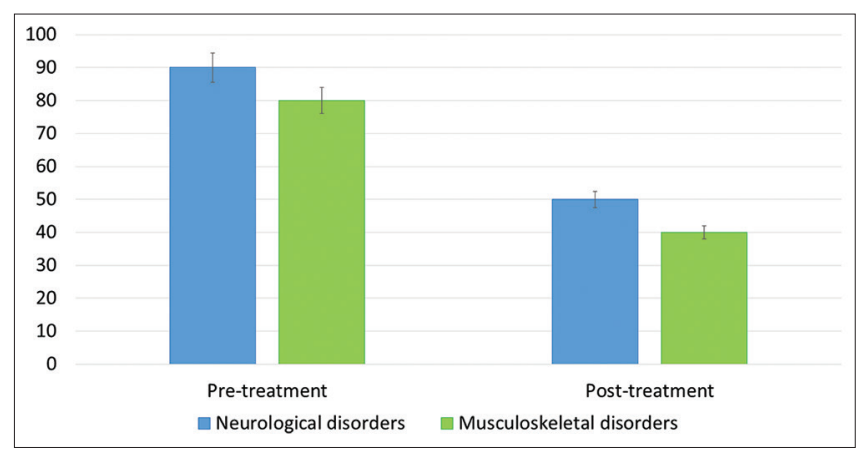

Figure 10: Significant improvement $(P<0.02)$ in the VAS pain score after 1-month FIT therapy 
As to the infrared putative therapeutic role, there have been a number of studies on various biological effects caused by $\mathrm{THz}$ waves. The majority of them deal with thermal and non-thermal effects due to $\mathrm{THz}$ irradiation ${ }^{10,11}$. For example, the influence of a high peak electric field of the $\mathrm{THz}$ wave has been investigated showing DNA remodelling effects ${ }^{12}$. Besides these studies, Feldman et al. have reported that the human sweat ducts in skin play a significant role in the interaction of $\mathrm{THz}$ wave with the skin ${ }^{13}$. In fact, it has been experimentally defined that the sweat duct can act as a low-Q-factor helical antenna when the helically-shaped sweat ducts are filled with the highly electrolytes concentrated conductive sweat fluid. The conductivity mechanism in this case is assigned to proton hopping though ordered aqueous H-bond networks, rather than ionic conductivity. These results have also been assessed by Yang et $\mathrm{a}^{14}$. Based upon the numerical modelling and experimental data, it was suggested that the sweat duct play a role in characterizing the phenomena of resonance behaviour. Similarly, Shaferstein et al. reported that the sweat ducts play a significant role in millimetre wave absorption, indicating possible thermal effects induced by such waves ${ }^{15}$. Recently, it has been investigated the absorbance of sub- $\mathrm{THz}$ radiation by human skin and demonstrated that the absorption of $\mathrm{THz}$ wave is governed by the topology for the skin and the sweat duct ${ }^{1}$. The helically structured sweat ducts play thus a significant role in the interaction with $\mathrm{THz}$ and the updated research trends on this issue have been addressed to channel characterization in human skin for body-centric nano-communication ${ }^{1}$ and the development of extremely high-power gyrotron operating in sub-terahertz frequency region and high data-rate terahertz communication links ${ }^{1}$.

Experimental studies conducted by the CNR Nano of Pisa compared emissivity of a patch covered by a powder of titanium metal dioxides and control titanium metal dioxides - free patch at the wavelength of $10.8 \mu \mathrm{m}$ and $37^{\circ}$ temperature in order to investigate if the addiction of titanium to the patch influences the patch-emitted energy. Results showed a greater value of emissivity $(+5 \%)$ with the titanium-covered patch compared to the control patch, and a $100 \%$ emissivity between 7 and $11 \mu \mathrm{m}$ wavelength, suggesting a strongest capacity to irradiate energy played by the titanium metal dioxides compared to a black body that was responsible to the therapeutic mechanism of action (personal communications). Since also our biological body structures naturally emit FIW probably, the addiction of titanium might contribute to the spread of the body energy and to modulate the physiological functions. However, we do not know if patch thickness might influence therapeutic response.
On this basis, the relationship between sweat glands density and the fit patches effects on the skin, and it's nociceptive receptors should be deeply investigated.

Apparently, the subcutaneous muscle mass does not seem to definitely interfere with the therapeutic response.

Actually quite often, the FIT PATCH mediated pain improvement reduced the NSAIDs or steroids administration schedule and it was not effective in cases of nerve mechanical compression (such as in herniated cervical or lumbar disks, articular conflicts nerve trapping like in the tennis elbow, etc.).

\section{CONCLUSIONS}

FIT therapy patches improved the symptoms probably interacting with the skin sweat glands whose ducts supposedly act as a low-Q-factor helical antenna $\mathrm{THz}$ irradiation amplifying the infrared signals emitted by the fit plasters. Completely marginal and irrelevant for therapeutic purposes, the hypothesised synergistic effect of the occlusive mechanism due to the adhesion on the surface of the skin, which inhibits the sweat sebaceous perspiration, increases the local thermal gradient; in fact, the heating induced by the occlusion is subjectively variable and acts strictly at the site of application, while the symptomatic effects of the application of FIT are also expressed remotely. The intrinsic chemical properties of the FIT patch are therefore characterized to absorb and concentrate infrared waves, generate endogenously, reverberating them in the affected district and accelerating functional recovery by dissipating cell and neurochemicallymediated inflammation.

\section{ACKNOWLEDGEMENTS}

We greatly acknowledge D.FENSTEC s.r.l., Altavilla Vicentina (VI), ITALY, for providing us FIT-PATCH.

\section{REFERENCES}

1. Tripathi SR, Ben Ishai $P$ and Kawase K. Frequency of the resonance of the human sweat duct in a normal mode of operation. Biomed Opt Express. 2018 Mar 1;9(3):1301-1308.

2. Adams JA, Galloway TS, Mondal D, Esteves SC and Mathews F. Effect of mobile telephones on sperm quality: a systematic review and meta-analysis. Environ Int. 2014 Sep;70:106-112.

3. Blank M and Goodman R. Electromagnetic fields stress living cells. Pathophysiology. 2009 Aug;16(2-3):71-78.

4. Darbandi M, Darbandi S, Agarwal A, Henkle R and Sadeghi MR. The Effects of Exposure to Low Frequency Electromagnetic Fields on Male Fertility. Altern Ther Health Med. 2017 Jun 23.

5. Hardell $L$ and Sage $C$. Biological effects from electromagnetic field exposure and public exposure standards. Biomed 
Pharmacother. 2008 Feb;62(2):104-109.

6. Di Cerbo A and Palmieri B. The economic impact of second opinion in pathology. Saudi Med J. 2012 Oct;33(10):1051-1052.

7. Palmieri B and lannitti T. The Web Babel syndrome. Patient Educ Couns. 2011 Nov;85(2):331-333.

8. Palmieri B, lannitti T, Capone S, Fistetto G and Arisi E. Second opinion clinic: is the Web Babel Syndrome treatable?. Clin Ter. 2011;162(6):575-583.

9. Ware JE and Sherbourne CD. The MOS 36-item short-form health survey (SF-36). I. Conceptual framework and item selection. Med Care. 1992 Jun;30(6):473-483.

10. Yang X, Zhao X, Yang K, Liu Y, Fu W and Luo Y. Biomedical Applications of Terahertz Spectroscopy and Imaging. Trends Biotechnol. 2016 Oct;34(10):810-824.

11. Romanenko S, Begley R, Harvey AR, Hool L and Wallace VP. The interaction between electromagnetic fields at megahertz, gigahertz and terahertz frequencies with cells, tissues and organisms: risks and potential. J R Soc Interface. 2017 Dec;14(137).

12. Titova LV, Ayesheshim AK, Golubov A, Fogen D, RodriguezJuarez $R$ and Hegmann FA. Intense $\mathrm{THz}$ pulses cause H2AX phosphorylation and activate DNA damage response in human skin tissue. Biomed Opt Express. 2013 Apr 1;4(4):559-68.

13. Feldman $Y$, Puzenko $A$, Ben Ishai $P$, Caduff $A$ and Agranat AJ. Human skin as arrays of helical antennas in the millimeter and submillimeter wave range. Phys Rev Lett. 2008 Mar 28;100(12):128102.

14. Yang B, Donnan RS, Zhou M and Kingravi AA. Reassessment of the electromagnetic reflection response of human skin at W-band. Opt Lett. 2011 Nov 1;36(21):4203-4205.

15. Shafirstein $G$ and Moros EG. Modelling millimetre wave propagation and absorption in a high resolution skin model: the effect of sweat glands. Phys Med Biol. 2011 Mar 7;56(5):1329-1339.

\section{Authors Contribution:}

CL - Concept and design of the study, manuscript preparation, statistically analyzed and interpreted, critical revision of the manuscript; BP - Concept and design of the study, critical revision of manuscript and review of the study; MV - reviewed the literature, helped in preparing first draft of manuscript, collected data.

Work attributed to:

Second Opinion Medical Network, Modena, Italy.

\section{Orcid ID:}

Prof. Beniamino Palmieri - (10) http://orcid.org/0000-0002-0871-138X

Dr. Maria Vadalà - (i) http://orcid.org/0000-0001-7873-5072

Dr. Carmen Laurino - (10) http://orcid.org/0000-0002-3020-2338

Source of Support: None, Conflict of Interest: The authors certify that there is no conflict of interest with any financial organization regarding the material discussed in the manuscript. The authors hereby certify that all work contained in this article is original. The authors claim full responsibility for the contents of the article. The article has been neither published elsewhere nor submitted for publication simultaneously. If accepted, the paper will not be published elsewhere in the same or similar form, in English or in any other language. All the authors contributed equally to this work. 